\title{
Transient postsurgical gastroparesis is accompanied by reversible changes of the cervical vagus nerve's morphology after neck dissection - an ultrasound study
}

\author{
Ömer Özçağlayan ${ }^{1}$, Tolga Ersözlü²
}

${ }^{1}$ Department of Radiology, ${ }^{2}$ Department of Otorhinolaryngology Tekirdağ Namık Kemal University, School of Medicine, Tekirdağ, Turkey

\begin{abstract}
Aims: During neck dissection (ND), the vagus nerve (VN) may be exposed to manipulation together with common carotid artery and internal jugular vein. The postsurgical gastroparesis was previous related to the VN injury. The aim of our study was to evaluate by ultrasound the VN changes in patients with unilateral and bilateral ND and to establish if there is a relationship between postoperative findings of VN and postsurgical gastroparesis. Material and methods: Seventeen patients in which $30 \mathrm{ND}$ (4 unilateral and 13 bilateral) were performed, were enrolled in the study. The VN's area and diameter were measured preoperative (baseline), one week (T1) and one month (T2) postoperative. Gastrointestinal symptoms were evaluated at T1 and T2 phases using the patient assessment of the upper gastrointestinal symptom severity index (PAGI-SYM). Results: There was a statistical difference between area and diameters of VN between T1 and baseline $(\mathrm{p}<0.001)$, and T1 and T2 phases ( $<<0.001)$, respectively. No statistical differences were detected at baseline and T2 phases in areas $(\mathrm{p}=0.934)$ and diameters $(\mathrm{p}>0.999)$. Gastrointestinal symptoms, found at the T1 phase regressed at T2 phase, were correlated with VN area and diameter changes $(\mathrm{p}<0.001)$. Conclusions: VN ultrasound clearly showed the transient dimensional changes of VN caused by manipulation in $\mathrm{ND}$, which may lead to temporary gastrointestinal symptoms due to reversible dysfunction of VN.
\end{abstract}

Keywords: neck dissection; ultrasound; gastroparesis; vagus nerve

\section{Introduction}

Neck dissection (ND) is an important component of diagnosis and treatment in head and neck cancers. The main goal of ND is to identify suspected lymph nodes and to extirpate pathological lymph nodes [1]. In addition to the lymph nodes, common carotid artery (CCA),

Received 03.10.2019 Accepted 02.12.2019

Med Ultrason

2020, Vol. 22, No 1, 26-30

Corresponding author: Ömer Özçağlayan, MD

Tekirdağ Namık Kemal University, School of Medicine, Department of Radiology

1 Namık Kemal Mah. Kampüs Cad, 59030 Süleymanpaşa, Tekirdağ, Turkey

E-mail: omer.ozcaglayan@gmail.com

Phone: 02822505001

Fax: 02822509928 internal jugular vein (IJV) and vagus nerve (VN) can be also affected during ND [2].

$\mathrm{VN}$ is the major nerve of the brain-gut axis [3]. It is well-known that vagotomy and drugs may cause VN dysfunction [4] but gastroparesis may be related to neurological disorders, such as Parkinson's disease [5].

Postsurgical gastroparesis (PSG) is defined as a syndrome characterized by postprandial nausea and vomiting, postoperative gastric atony, distention and upper gastrointestinal discomfort without mechanical gastric outlet obstruction [6]. PSG is a phenomenon observed in upper abdominal surgery, especially in vagotomy and vagus injury [7].

$\mathrm{VN}$ is particularly exposed to manipulation during $\mathrm{ND}$, upper gastrointestinal changes being observed in patients in the first month after ND [8]. PSG like symptoms after ND might depend on VN manipulation. 
The aim of this study was to evaluate the possible morphological changes of $\mathrm{VN}$ using ultrasound examination in patients undergoing ND and to evaluate the relationship between these changes and potential PSG symptoms.

\section{Materials and methods}

\section{Study design and population selection}

The study was approved by the local Ethics Committee. Seventeen patients with head and neck malign tumors who underwent ND between August 2018 and April 2019 in Otorhinolaryngology clinic were enrolled. Sign informed consent was obtained from all patients. The criteria for inclusion were age over 18 years and the presence of a malignant mass in the head and neck with indication for ND. Exclusion criteria were diabetes mellitus, Parkinson's disease, VN mass and previous radiotherapy (RT) to the neck. All patients underwent modified radical neck dissection which included lymph node levels I, II, III and IV. The carotid sheath fascia was incised at III and IV level in the neck region. Once VN was identified, the fascia overlying the IJV was peeled off (fig 1).

\section{Vagus nerve ultrasound protocol}

VN ultrasound was performed by the same examiner using Toshiba ${ }^{\mathrm{TM}}$ Aplio ${ }^{\mathrm{TM}} \mathrm{XG}$ ultrasound device with a 6-12 MHz linear probe. At the level of the thyroid gland on the axial ultrasound image, CCA and IJV were found. At neck level III and IV, lower cervical VN was observed between these anatomic structures in the vagina caroticum as a hypoechogenic round image and its continuity was depicted according to the literature $[9,10]$. After that, the probe was rotated with $90^{\circ}$ and the $\mathrm{VN}$ was examined

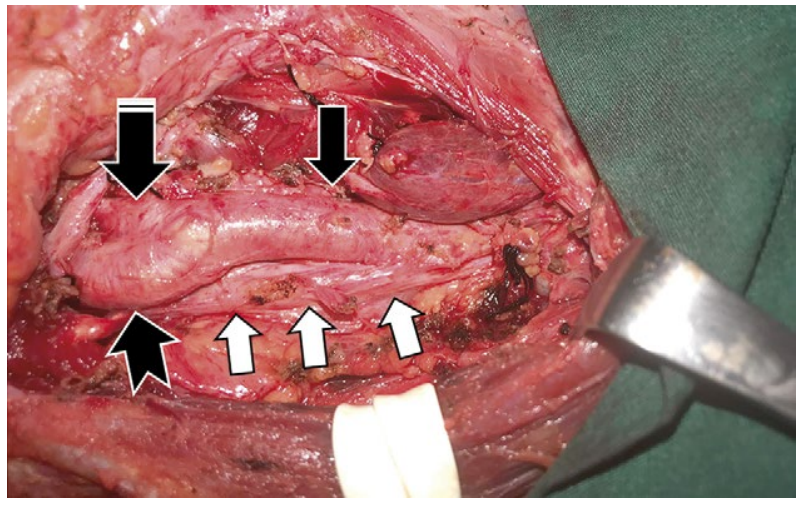

Fig 1. Appearance of neck dissection following removal of the IJV. VN (white arrows) can clearly be seen behind the CCA (black arrow), ECA (black stripe arrow) and ICA (black tailed arrow). IJV, Internal Jugulary Vein; VN, Vagus Nerve; CCA, Common Carotid Artery; ECA, External Carotid Artery; ICA, Internal Carotid Artery. in longitudinal axis. The VN area was measured in the axial plane and its diameter in longitudinal axis (fig 2).

The VNs were evaluated before ND (baseline), 1 week (T1) and 1 month postoperative (T2). Changes of diameter, area and echogenicity in VN were noted.

\section{Evaluation of gastrointestinal system}

All patients were examined by an experienced specialist in gastrointestinal motility disorders using a detailed medical history, physical examination and comprehensive metabolic panel to exclude a pre-existing gastrointestinal system disorder and possible non-PSG gastroparesis. The patients were evaluated with the "patient assessment of upper gastrointestinal symptom severity index" (PAGI-SYM) test to evaluate the gastrointestinal system at 1 week (T1) and 1 month (T2) postoperative phases. During the PAGI-SYM evaluation, day heartburn, regurgitation or reflux, nausea, upper abdominal pain, stomach fullness, bloating, heartburn (lying down), retching, vomiting and upper abdominal discomfort symptoms were investigated as described in the literature at $\mathrm{T} 1$ and $\mathrm{T} 2$ phases and were scored from 0 to 5 according to symptom severity $[11,12]$.

\section{Statistical analysis}

Data were analyzed using IBM SPSS Statistics 17.0 (IBM Corporation, Armonk, NY, USA). The ShapiroWilk test was used to determine whether the distribution of continuous numerical variables was close to normal. In the descriptive statistics, continuous numerical variables were expressed as the mean \pm standard deviation, while categorical variables were shown as the number of cases and percentages (\%).

The statistical relationship of the mean vagus area and vagus diameter between the evaluation periods was

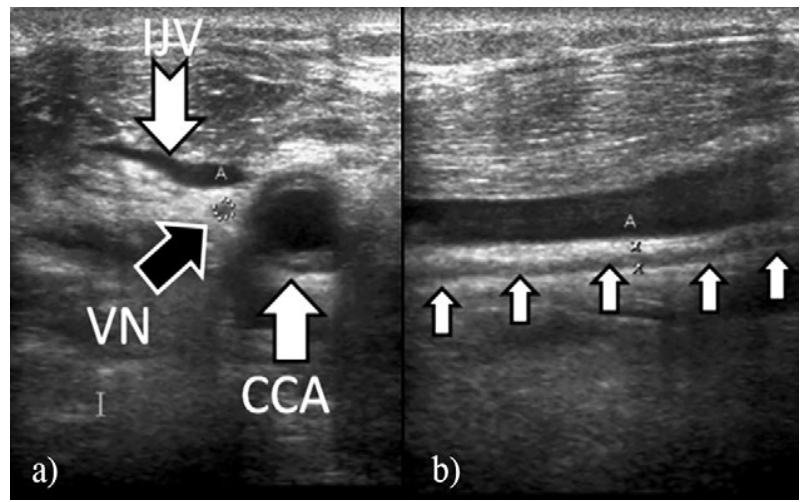

Fig 2. a) Axial neck ultrasound shows the VN (black arrow) as a hypoechoic round image between CCA (white arrow) and IJV (white tailed arrow); b) Longitudinal neck ultrasound shows the longitudinal trace of VN (white arrows) behind the IJV. VN, Vagus Nerve; CCA, Common Carotid Artery; IJV, Internal Jugulary Vein. 
evaluated using the Lambda test of Wilks with a Variance Analysis in repeated measurements. For unilateral dissection cases, the dependent t-test was used to determine whether there was a statistically significant difference between the dissected and the non-dissected sides of the neck in terms of the mean vagus area and the vagus diameter. The statistical significance between PAGI-SYM values at $\mathrm{T} 1$ and $\mathrm{T} 2$ phases was determined by the Wilcoxon signed rank test. A p value of $<0.05$ was accepted to be statistically significant.

\section{Results}

The mean age of the 17 patients was $59.0 \pm 13.6$ (range: $36-86$ years) and 14 patients $(82.4 \%)$ were men. Four $(23.5 \%)$ of the patients underwent unilateral ND and 13 (76.5\%) bilateral ND. There were statistically significant differences in the mean $\mathrm{VN}$ areas and diameters between the baseline and $\mathrm{T} 1$ phases $(\mathrm{p}<0.001)$ and between $\mathrm{T} 1$ and $\mathrm{T} 2$ phases $(\mathrm{p}<0.001)$, respectively. There were no statistically significant differences between mean VN areas $(\mathrm{p}=0.934)$ and diameters $(\mathrm{p}>0.999)$ at the baseline and T2 phases (Table I) (fig 3). In patient with unilateral $\mathrm{ND}$, areas and diameters of $\mathrm{VN}$ modified only in the dis-

Table I. Vagus nerve area and diameter according to follow-up time

\begin{tabular}{lll}
\hline & VN area $\left(\mathbf{m m}^{\mathbf{2}}\right)$ & VN diameter $(\mathbf{m m})$ \\
\hline Baseline & $2.09 \pm 0.62^{\mathrm{a}}$ & $1.70 \pm 0.25^{\mathrm{a}}$ \\
T1 phase & $4.35 \pm 1.41^{\mathrm{a}, \mathrm{b}}$ & $2.36 \pm 0.38^{\mathrm{a}, \mathrm{b}}$ \\
T2 phase & $2.23 \pm 0.77^{\mathrm{b}}$ & $1.76 \pm 0.34^{\mathrm{b}}$ \\
$\mathrm{p}$-value $\dagger$ & $<\mathbf{0 . 0 0 1}$ & $<\mathbf{0 . 0 0 1}$ \\
\hline
\end{tabular}

Data shown as mean \pm standard deviation. $\dagger$ Analysis of variance in repeated measures, Lambda test of Wilks; a: The difference between baseline and T1 phase was statistically significant $(\mathrm{p}<0.001)$; $\mathrm{b}$ : The difference between T1 phase and T2 phase was statistically significant $(\mathrm{p}<0.001)$. VN: Vagus Nerve.

Table II. Vagus nerve areas and diameters of dissection side and contralateral side in unilateral ND cases

\begin{tabular}{llll}
\hline & $\begin{array}{l}\text { Dissection } \\
\text { side }\end{array}$ & $\begin{array}{l}\text { Non-dissected } \\
\text { side }\end{array}$ & p-value $\dagger$ \\
\hline VN area & & & \\
Baseline & $3.25 \pm 0.50$ & $2.50 \pm 1.29$ & 0.319 \\
T1 phase & $6.50 \pm 2.08$ & $2.75 \pm 0.96$ & $\mathbf{0 . 0 3 6}$ \\
T2 phase & $3.25 \pm 0.96$ & $2.75 \pm 0.96$ & 0.604 \\
VN diameter & & & \\
Baseline & $2.20 \pm 0.22$ & $1.77 \pm 0.17$ & 0.099 \\
T1 phase & $3.00 \pm 0.29$ & $2.02 \pm 0.39$ & $\mathbf{0 . 0 2 9}$ \\
T2 phase & $2.05 \pm 0.19$ & $2.02 \pm 0.40$ & 0.934 \\
\hline
\end{tabular}

Data shown as mean \pm standard deviation. $\dagger$ Dependent $t$ test. VN: Vagus Nerve, ND: Neck dissection sected side (Table II). All the gastrointestinal symptoms scored from 0 to 5 in PAGI-SYM evaluation had higher scores in $\mathrm{T} 1$ comparing with T2 phase $(\mathrm{p}<0.001)$ (Table III).

\section{Discussion}

Previous published studies have focused on VN injuries in ND but the transient nerve dysfunction due to manipulation without nerve injury was not discussed [13]. We clearly demonstrated that in patients undergoing ND, the $\mathrm{VN}$ increases in diameter and cross sectional area in the first postoperative week due to accompanying edema. In these cases, without injury in the $\mathrm{VN}$, the pathologic

Table III. Patient assessment of upper gastrointestinal symptom severity index (PAGI-SYM) scores of the post-operative first week (T1) and postoperative first month (T2) phases of cases

\begin{tabular}{lll}
\hline & & p-value $\dagger$ \\
\hline Heartburn (during day) & & $<\mathbf{0 . 0 0 1}$ \\
T1 phase & $3.29 \pm 0.69(2-5)$ & \\
T2 phase & $0.76 \pm 0.56(0-2)$ & \\
Regurgitation or reflux & & $\mathbf{0 . 0 0 4}$ \\
T1 phase & $3.00 \pm 0.94(1-5)$ & \\
T2 phase & $1.53 \pm 0.87(0-3)$ & \\
Nausea & & $\mathbf{0 . 0 0 2}$ \\
T1 phase & $2.76 \pm 0.97(1-4)$ & \\
T2 phase & $1.35 \pm 0.86(0-3)$ & \\
Upper abdominal pain & & $\mathbf{0 . 0 0 3}$ \\
T1 phase & $2.06 \pm 1.14(0-4)$ & \\
T2 phase & $0.88 \pm 0.78(0-2)$ & \\
Stomach fullness & & $\mathbf{0 . 0 0 3}$ \\
T1 phase & $2.24 \pm 0.83(1-3)$ & \\
T2 phase & $1.18 \pm 0.95(0-3)$ & \\
Bloating & & $\mathbf{0 . 0 1 0}$ \\
T1 phase & & \\
T2 phase & & \\
Heartburn (lying down) & & \\
T1 phase & $3.35 \pm 1.11(0-4)$ & \\
T2 phase & & \\
Retching & & \\
T1 phase & & \\
T2 phase & & \\
Vomiting & & \\
T1 phase & & \\
T2 phase & & \\
Upper abdominal discomfort & & \\
T1 phase & & \\
T2 phase & & \\
\hline
\end{tabular}

Data shown as mean \pm standard deviation (minimum-maximum). $\dagger$ Wilcoxon signed rank test 


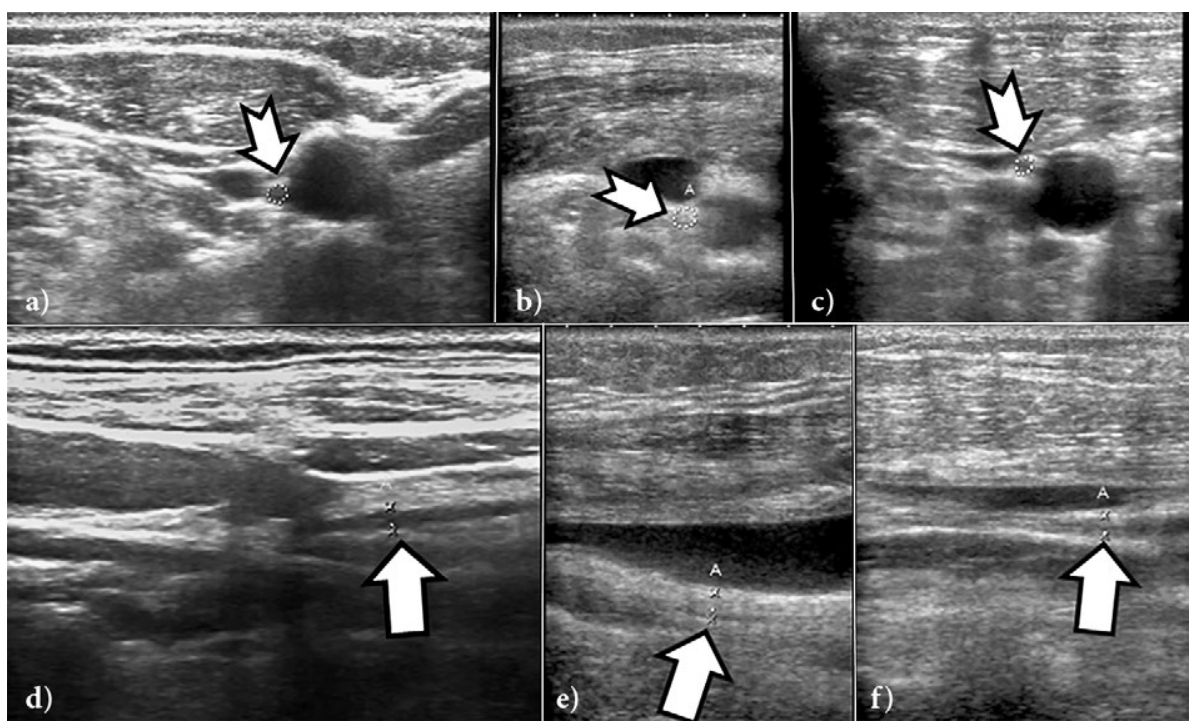

Fig 3. Axial ultrasound shows the area of the VN at a) baseline $\left(2 \mathrm{~mm}^{2}\right)$, b) T1 $\left(6 \mathrm{~mm}^{2}\right)$ and c) T2 $\left(2 \mathrm{~mm}^{2}\right)$ phases (white tailed arrows). Ultrasound also shows the increase of echogenicity of $\mathrm{VN}$ due to edema at $\mathrm{T} 1$ phase. Longitudinal ultrasound shows the diameter of the $\mathrm{VN}$ at d) baseline $(1.6 \mathrm{~mm}), \mathrm{e}) \mathrm{T} 1(3.1 \mathrm{~mm})$ and f) T2 $(1.8 \mathrm{~mm})$ phases (white arrows). VN, Vagus Nerve.

findings resolved in the first month after operation. Our study also depicted that there were no changes of $\mathrm{VN}$ dimensions on the non-dissecting side, and significant changes on the dissection side due to VN manipulation at unilateral neck dissection.

We demonstrated that this reversible increase in VN dimensions correlated with the gastrointestinal symptoms associated with reversible PSG and the symptoms regressed due to the resolution of VN edema. The manipulation of the $\mathrm{VN}$ during $\mathrm{ND}$ is the main cause for the reversible edema, as we established in patients with unilateral ND.

ND is increasingly performed as an important component of head and neck surgery [14]. Following neck surgery, VN injuries can be viewed as surgical complications. VN injuries usually manifest due to IJV ligations [15]. The evaluation of $\mathrm{VN}$ in an anterior triangle is especially important for VN injuries [16]. Polistena et al [17] reported that in 1765 patients who underwent thyroidectomy and neck dissection, only one patient $(0.14 \%)$ had VN injury. Depending on the level of VN injury, vocal cord paralysis, dysphagia and aspirations can appear $[1,18]$.

In the literature, the effects of ND on CCA and IJV have been discussed [19-21]. There are also studies about the patency of IJV in ND [22,23]. In these studies, the lumen, flow velocity, and thrombus presence of IJV after ND were evaluated. There are also studies about morphological changes and function of VN. Grimm et al showed the enlargement of $\mathrm{VN}$ in the acute phase of the Guillain-
Barré syndrome [24] and Pelz et al depicted the relationship between heart rate variabilities and VN area using ultrasound [25].

Gastroparesis, an entity that develops due to VN dysfunction, can occur after surgery (PSG) or in patients with diabetes mellitus, Parkinson's disease, multiple sclerosis or connective tissue diseases [26,27]. PSG is the third most common type of gastroparesis and accounts for approximately $3-17 \%$ of all gastroparesis cases. Gastrectomies, vagotomies and thoracic surgery are the most frequent situation associated with PSG [6]. Particularly after lung transplantation, PSG may become manifest and due to gastroesophageal reflux, microaspirations to the lung and consequently bronchiolitis may occur [28]. The "vagal nerve integrity test" can be used to determine whether gastroparesis is secondary to VN injury or dysfunction [29]. We found that PSG-like upper gastrointestinal system symptoms occurred in the postoperative first week and regressed in the fourth week. The coincidence of these symptoms with edema due to the manipulation of $\mathrm{VN}$ and consequently with the increase in VN diameter and area measured confirmed the initial hypothesis.

In this study, the small number of cases was the main limitation. Further studies with more patients would provide a broader understanding of $\mathrm{VN}$ dysfunction and related gastrointestinal symptoms in ND. The lack of intra and interobserver reliability, absence of comparison with other imaging techniques and comparison with the level of dissection are other limitations of the study. 
In conclusion, ultrasound is a useful diagnostic imaging technique for the postsurgical screening of neuronal and vascular structures after ND. The reversible edema in $\mathrm{VN}$ producing a temporary increase in the dimensions of $\mathrm{VN}$ is responsible for reversible upper gastrointestinal symptoms.

\section{Conflict of interest: None}

\section{References}

1. Dedivitis RA, Guimarães AV, Pfuetzenreiter EG Jr, Castro MA. Neck dissection complications. Braz J Otorhinolaryngol 2011;77:65-69.

2. Kerawala CJ, Heliotos M. Prevention of complications in neck dissection. Head Neck Oncol 2009;1:35.

3. Bonaz B, Sinniger V, Pellissier S. The Vagus Nerve in the Neuro-Immune Axis: Implications in the Pathology of the Gastrointestinal Tract. Front Immunol 2017;8:1452.

4. Stakenborg N, Di Giovangiulio M, Boeckxstaens GE, Matteoli $\mathrm{G}$. The versatile role of the vagus nerve in the gastrointestinal tract. EMJ Gastroenterol 2013;1:106-114.

5. Pelz JO, Belau E, Fricke C, Classen J, Weise D. Axonal Degeneration of the Vagus Nerve in Parkinson's Disease-A High-Resolution Ultrasound Study. Front Neurol 2018;9:951.

6. Dong K, Yu XJ, Li B, Wen GA, Xiong W, Guan QL. Advances in mechanisms of postsurgical gastroparesis syndrome and its diagnosis and treatment. Chin J Dig Dis 2006; 7:76-82.

7. Shafi MA, Pasricha PJ. Post-surgical and obstructive gastroparesis. Curr Gastroenterol Rep 2007;9:280-285.

8. Jones E, Lund VJ, Howard DJ, Greenberg MP, McCarthy M. Quality of life of patients treated surgically for head and neck cancer. J Laryngol Otol 1992;106:238242.

9. Chen HH, Chen TC, Yang TL, Wang CP. Transcutaneous Sonography for Detection of the Cervical Vagus Nerve. Ear Nose Throat J 2019:145561319875432.

10. Pelz JO, Belau E, Henn P, Hammer N, Classen J, Weise D. Sonographic evaluation of the vagus nerves: Protocol, reference values, and side-to-side differences. Muscle Nerve 2018;57:766-771.

11. Rentz AM, Kahrilas P, Stanghellini V, et al. Development and psychometric evaluation of the patient assessment of upper gastrointestinal symptom severity index (PAGISYM) in patients with upper gastrointestinal disorders. Qual Life Res 2004;13:1737-1749.

12. Jehangir A, Parkman HP. Rome IV Diagnostic Questionnaire Complements Patient Assessment of Gastrointestinal Symptoms for Patients with Gastroparesis Symptoms. Dig Dis Sci 2018;63:2231-2243.

13. Kakisis JD, Antonopoulos CN, Mantas G, Moulakakis KG, Sfyroeras G, Geroulakos G. Cranial Nerve Injury After Carotid Endarterectomy: Incidence, Risk Factors, and Time Trends. Eur J Vasc Endovasc Surg 2017;53:320-335.
14. Kim EY, Eisele DW, Goldberg AN, Maselli J, Kezirian EJ. Neck dissections in the United States from 2000 to 2006: volume, indications, and regionalization. Head Neck 2011;33:768-773.

15. Genden EM, Ferlito A, Shaha AR, et al. Complications of neck dissection. Acta Otolaryngol 2003;123:795-801.

16. Chintamani. Ten Commandments of Safe and Optimum Neck Dissections for Cancer. Indian J Surg 2015;77:85-91.

17. Polistena A, Monacelli M, Lucchini R, et al. Surgical morbidity of cervical lymphadenectomy for thyroid cancer: A retrospective cohort study over 25 years. Int J Surg 2015;21:128-134.

18. Cece JA, Lawson W, Biller HF, Eden AR, Parisier SC. Complications in the management of large glomus jugulare tumors. Laryngoscope 1987;97:152-157.

19. Cramer JD, Patel UA, Maas MB, Samant S, Smith SS. Is Neck Dissection Associated with an Increased Risk of Postoperative Stroke? Otolaryngol Head Neck Surg 2017; 157:226-232.

20. Kamizono K, Ejima M, Taura M, Masuda M. Internal jugular vein reconstruction: application of conventional type A and novel type K methods. J Laryngol Otol 2011;125:643648.

21. Chung MK, Choi J, Lee JK, Jeong JI, Lee WY, Jeong HS. Preservation of the external jugular venous drainage system in neck dissection. Otolaryngol Head Neck Surg 2009;141:730-736.

22. Veyseller B, Aksoy F, Açikalin M, Yildirim YS, Ivgin Bayraktar FG, Demirhan H. Assessment of internal jugular vein flow and patency with power duplex Doppler ultrasonography after functional neck dissection. Kulak Burun Bogaz Ihtis Derg 2010;20:38-43.

23. Prim MP, de Diego JI, Fernández-Zubillaga A, Garcia-Raya P, Madero R, Gavilan J. Patency and flow of the internal jugular vein after functional neck dissection. Laryngoscope 2000;110:47-50.

24. Grimm A, Decard BF, Axer H. Ultrasonography of the peripheral nervous system in the early stage of Guillain-Barré syndrome. J Peripher Nerv Syst 2014;19:234-241.

25. Pelz JO, Belau E, Menze I, Woost TB, Classen J, Weise D. Correlation between sonographic morphology and function of the cervical vagus nerves. Auton Neurosci 2019;220:102552.

26. Quigley EM. Other forms of gastroparesis: postsurgical, Parkinson, other neurologic diseases, connective tissue disorders. Gastroenterol Clin North Am 2015;44:6981.

27. Bielefeldt K. Gastroparesis: concepts, controversies, and challenges. Scientifica (Cairo) 2012;2012:424802.

28. Berkowitz N, Schulman LL, McGregor C, Markowitz D. Gastroparesis after lung transplantation. Potential role in postoperative respiratory complications. Chest 1995;108:1602-1607.

29. Yolsuriyanwong K, Marcotte E, Venu M, Chand B. Impact of vagus nerve integrity testing on surgical management in patients with previous operations with potential risk of vagal injury. Surg Endosc 2019;33:2620-2628. 